\title{
Thermal Characterization of Electrically Injected Thin-Film InGaAsP Microdisk Lasers on Si
}

\author{
Joris Van Campenhout, Student Member, IEEE, Pedro Rojo-Romeo, Dries Van Thourhout, Member, IEEE, \\ Christian Seassal, Philippe Regreny, Lea Di Cioccio, Jean-Marc Fedeli, and Roel Baets, Fellow, IEEE
}

\begin{abstract}
We have performed a numerical and experimental analysis of the thermal behavior of electrically injected microdisk lasers that are defined in an InGaAsP-based thin film bonded on top of a silicon wafer. Both the turn-on as well as the pulsed-regime temperature evolution in the lasing region was simulated using the finite-element method. The simulation results are in good agreement with experimental data, which was extracted from the broadening of the time-averaged emission spectra. Lasing at room temperature was only possible in pulsed regime due to the high thermal resistance $(10 \mathrm{~K} / \mathrm{mW})$. Some strategies to decrease the thermal resistance of the microdisk lasers are proposed and discussed.
\end{abstract}

Index Terms-Heterogeneous integration, InGaAsP, integrated optics, microdisk laser, $\mathrm{Si}$, thermal characterization.

\section{INTRODUCTION}

$\mathbf{M}$ ICRODISK lasers have attracted much interest lately, mostly due to their potential role as very compact light sources with low power consumption in large-scale photonic integrated circuits. Several authors have reported electrically injected lasing in microdisk structures that are supported by a pedestal, with some having lasing thresholds of well below $100 \mu \mathrm{A}$ [1]-[4]. The collection of the laser light can be done by means of evanescent coupling to a passive waveguide, as demonstrated in [5]. Our work focuses on the integration of these III-V microdisk lasers on an Si platform. This approach facilitates integration not only with silicon electronics but also with silicon photonics. Indeed, because of the transparency of $\mathrm{Si}$ at the telecommunications wavelengths 1.3 and $1.55 \mu \mathrm{m}$, and the fact that complementary metal-oxide-semiconductor technology can be used in the fabrication of photonic compo-

Manuscript received September 11, 2006; revised February 16, 2007. This work was supported in part by the EU-funded IST Project PICMOS (FP6-2002-IST-1-002131), by the Belgian IAP-Photon Network, and by the IST-ePIXnet network of excellence. The work of J. Van Campenhout was supported by the Research Foundation-Flanders (FWO-Vlaanderen) through a doctoral fellowship.

J. Van Campenhout, D. Van Thourhout, and R. Baets are with the Photonics Research Group, Department of Information Technology (INTEC), Ghent University-Interuniversity Microelectronics Centre, 9000 Ghent, Belgium (e-mail: Joris.VanCampenhout@intec.UGent.be).

P. Rojo-Romeo, C. Seassal, and P. Regreny are with the Institut des Nanotechnologies de Lyon, Université de Lyon, INL-UMR5270, CNRS, Ecole Centrale de Lyon, F-69134 Ecully, France.

L. Di Cioccio and J.-M. Fedeli are with the Commissariat a l'Energie Atomique/Laboratoire d'Electronique de Technologie de l'Information, 38054 Grenoble, France.

Color versions of one or more of the figures in this paper are available online at http://ieeexplore.ieee.org.

Digital Object Identifier 10.1109/JLT.2007.895552

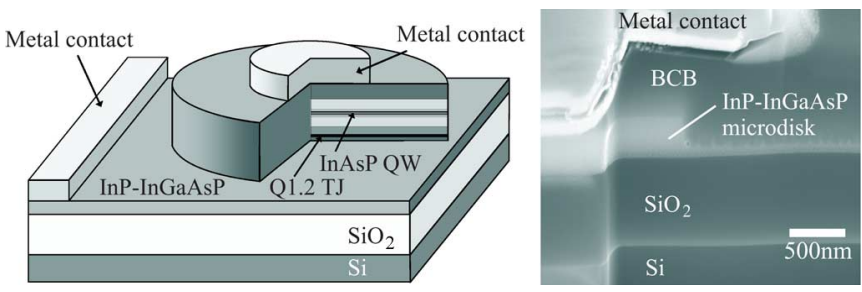

Fig. 1. (Left) Schematic representation of the microdisk laser structure and (Right) scanning-electron-microscope photo of the edge of the microdisk laser in cross section.

nents in silicon-on-insulator (SOI) [6], silicon has emerged as a promising platform for photonic functions. A big obstacle, however, is the poor light generation efficiency of silicon. This has been addressed by using the Raman effect [7], [8]. However, the optical gain in Si remains relatively low compared with III-V materials, resulting in very long laser structures. In our approach, the integration of compact active optoelectronic components on a silicon platform is done by bonding a thin III-V film on top of it. Optically pumped lasing in microdisk lasers that are integrated on an Si wafer has already been demonstrated [9], as well as their optical coupling to an underlying SOI waveguide [10]. Another demonstration of a heterogeneous approach can be found in [11]. Recently, we have demonstrated the electrically injected lasing operation of a microdisk laser that is integrated on an Si wafer [12]. Only pulsed operation was possible at room temperature due to serious self-heating. For most practical applications, efficient continuous-wave (CW) operation at room temperature (and above) will be needed, together with a minimal thermal rollover. Therefore, this selfheating should be strongly reduced. A first step toward this goal is a thermal analysis of these integrated thin-film microdisk lasers, which is presented in this paper.

\section{Microdisk Laser Structure AND LASING CHARACTERISTICS}

A schematic representation of the microdisk laser structure is given in Fig. 1. Microdisks with diameter $D$ in the range of 4-9 $\mu \mathrm{m}$ were etched into an InGaAsP-based thin film that is molecularly bonded on top of a $\mathrm{Si}$ wafer, with an intermediate 1- $\mu \mathrm{m}$-thick $\mathrm{SiO}_{2}$ bonding layer, that is deposited with plasma-enhanced chemical vapor deposition (PECVD). The active layer contains three InAsP quantum wells that are embedded in undoped Q1.2-barrier layers and is surrounded by an $\mathrm{n}+\mathrm{InP}$ contact layer on top and a p+ InP layer below. In order to avoid high optical absorption in p-type contact layers, 


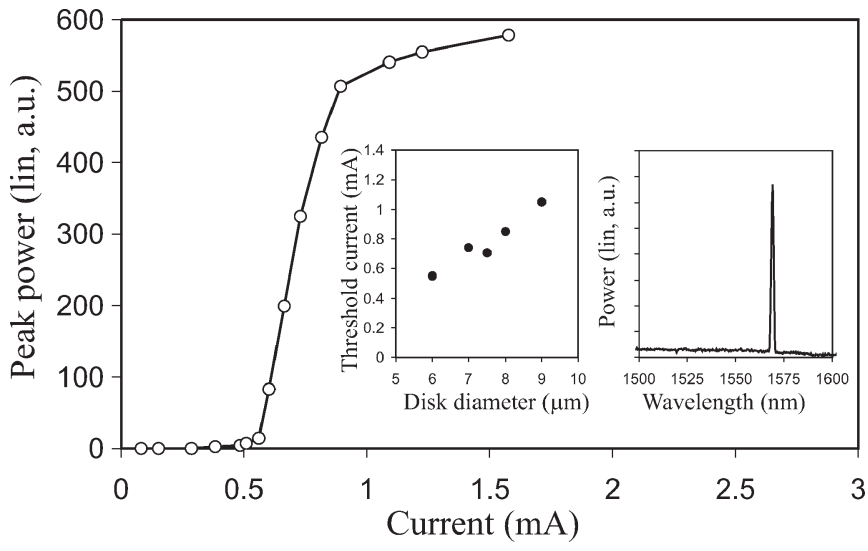

Fig. 2. Typical pulsed PI curve for $D=6 \mu \mathrm{m}$, with $T=20{ }^{\circ} \mathrm{C}$ (100-ns pulses, $3-\mu$ s period). Left inset: Threshold current as a function of disk diameter. Right inset: Lasing spectrum for $D=6 \mu \mathrm{m}$ and $I=0.75 \mathrm{~mA}$.

a reverse-biased Q1.2 tunnel junction was implemented, in combination with another $\mathrm{n}+$ contact layer. The total III-V film thickness is $480 \mathrm{~nm}$. The microdisk etch is incomplete, leaving an 80 -nm-thick lateral contact layer at the bottom of the disk. Before depositing the metal contacts, the structure is covered with a benzocyclobutene (BCB) film, in which contact windows are etched. Since the fundamental laser modes of a microdisk are whispering gallery modes that are located at the edges of the disk, the top contact window is only etched open at the center of the disk to avoid excess optical absorption due to the top metal. The bottom metal contact is placed a few micrometers from the disk. A cross section of a fully processed microdisk laser is also depicted in Fig. 1. A more detailed overview of the laser structure and fabrication aspects can be found in [12]. For the electroluminescence measurements, a variable voltage was applied over the electrodes, and a fraction of the emitted light was collected by a multimode fiber and fed into a spectrum analyzer, with a minimum spectral resolution of $0.2 \mathrm{~nm}$. Fig. 2 shows the peak power versus current (PI) characteristic at room temperature in the pulsed regime for a disk with $D=$ $6 \mu \mathrm{m}$. The PI curve reveals a clear threshold current $I_{\mathrm{th}}$ of $0.55 \mathrm{~mA}\left(1.95 \mathrm{kA} / \mathrm{cm}^{2}\right)$. The inset at the right shows the emission spectrum for $I=0.75 \mathrm{~mA}$, with a clear laser peak at $1570 \mathrm{~nm}$. The left inset shows the threshold currents for disks with $D$ ranging from 6 to $9 \mu \mathrm{m}$. For $D<6 \mu \mathrm{m}$, the BCB top contact window could not be etched open. The threshold voltage varies between 5 and $7 \mathrm{~V}$. This high value and its variation are due to a nonoptimal tunnel junction and nonoptimal metal contacts. Indeed, for some devices, the threshold voltage was reduced after sending a large current through the structure, most probably due to self-annealing of the metal contacts. In a second set of measurements, the temperature of the $\mathrm{Si}$ substrate was elevated from $10{ }^{\circ} \mathrm{C}$ up to $70{ }^{\circ} \mathrm{C}$ while recording the pulsed PI curve and the emission spectrum at each temperature. The results for $D=6 \mu \mathrm{m}$ can be found in Fig. 3. Lasing was observed up to $70{ }^{\circ} \mathrm{C}$, with $I_{\mathrm{th}}=1.05 \mathrm{~mA}$ and a strongly reduced laser efficiency. The temperature dependence of the lasing threshold can roughly be fitted as $I_{\mathrm{th}}=I_{0} \exp \left(T / T_{0}\right)$, with $I_{0}=0.39 \mathrm{~mA}$ and $T_{0}=71 \mathrm{~K}$. The emission wavelength $\lambda_{r}$ shifts with temperature at a rate of $d \lambda_{r} / d T=86 \mathrm{pm} / \mathrm{K}$. This value is lower than the expected $100 \mathrm{pm} / \mathrm{K}$ for InP-based lasers

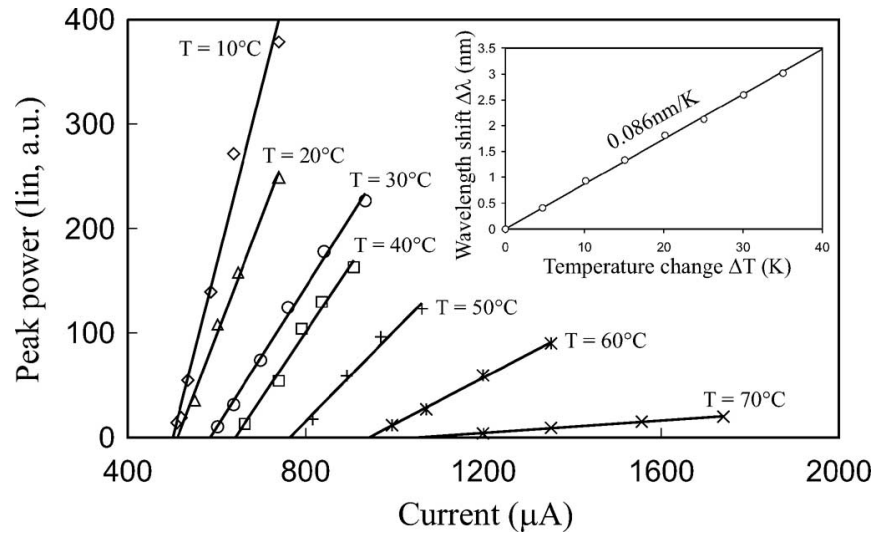

Fig. 3. Temperature dependence of the PI curve for $D=6 \mu \mathrm{m}$ (100-ns pulses, $3-\mu$ s period). Inset: Temperature dependence of the lasing wavelength.

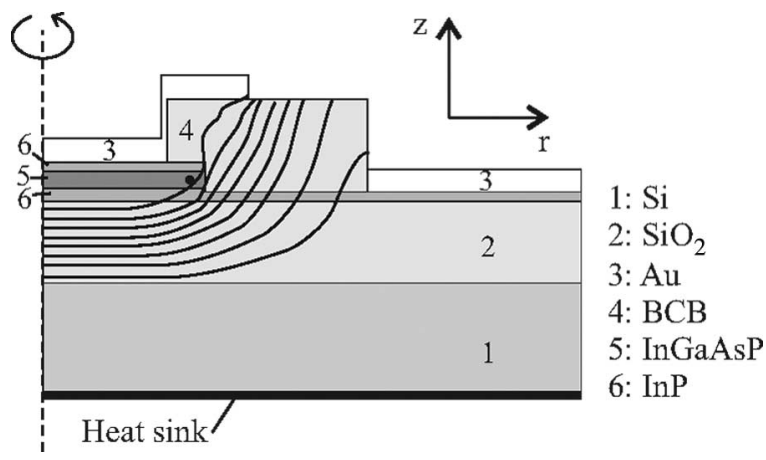

Fig. 4. Geometry used for thermal simulations, together with a typical temperature distribution. Temperatures are recorded in (black dot) the lasing region.

but is very close to the $90 \mathrm{pm} / \mathrm{K}$ that is reported in [3] for BCB-covered microdisk lasers. The reduction in wavelength shift is attributed to the negative temperature dependence of the refractive index of BCB. For larger disk diameters, we measured wavelength shifts of up to $95 \mathrm{pm} / \mathrm{K}$.

\section{Thermal Simulation}

In order to model the thermal behavior of the microdisk lasers, the heat equation should be solved as

$$
\rho c \frac{\partial T}{\partial t}=q_{v}+\nabla \cdot(\kappa \nabla T)
$$

where $\rho$ is the density, $c$ is the specific heat, $T(\mathbf{r}, t)$ is the temperature, $t$ is the time, $q_{v}$ is the heat dissipation per unit volume, and $\kappa$ is the thermal conductivity. Since the complete microdisk lasing structure has cylindrical symmetry (except for the metal pads), we chose to solve this problem in cylindrical coordinates, i.e.,

$$
r \rho c \frac{\partial T}{\partial t}=q_{v} r+\frac{\partial}{\partial r}\left(\kappa r \frac{\partial T}{\partial r}\right)+\frac{\partial}{\partial z}\left(\kappa r \frac{\partial T}{\partial z}\right)
$$

with $r$ and $z$ being the cylindrical coordinates. Coordinate $\theta$ does not appear in the equation due to symmetry. The laser structure that was used in the thermal model is depicted in Fig. 4. The geometry parameters were taken from the cross 
TABLE I

Material Parameters USED IN THE Thermal Simulation

\begin{tabular}{lccc}
\hline Material & $\begin{array}{c}\rho \\
\left(\mathrm{g} \mathrm{cm}^{-3}\right)\end{array}$ & $\begin{array}{c}\kappa \\
\left(\mathrm{Wm}^{-1} \mathrm{~K}^{-1}\right)\end{array}$ & $\begin{array}{c}c \\
\left(\mathrm{Jg}^{-1} \mathrm{~K}^{-1}\right)\end{array}$ \\
\hline $\mathrm{Si}$ & 2.3 & 130 & 0.70 \\
$\mathrm{SiO}_{2}$ & 2.2 & $0.8-1.4(1.27)$ & 0.74 \\
$\mathrm{Au}$ & 19.3 & 150 & 0.13 \\
$\mathrm{BCB}$ & 1.0 & 0.3 & 2.18 \\
$\mathrm{InGaAsP}$ & 4.8 & 6 & 0.31 \\
$\mathrm{InP}$ & 4.8 & 40 & 0.31 \\
$\mathrm{Al}_{2} \mathrm{O}_{3}(\mathrm{c})$ & 3.9 & 36 & 0.93 \\
$\mathrm{Al}_{2} \mathrm{O}_{3}(\mathrm{a})$ & 3.9 & 2 & 0.93 \\
$\mathrm{MgO}_{(\mu \mathrm{c})}$ & 3.6 & 4 & 0.88 \\
\hline
\end{tabular}

section in Fig. 1. A commercial finite-element tool was used to solve (2). Neumann boundary conditions were set on all the boundaries of the structure, except for the bottom boundary, which is kept at a fixed temperature (Dirichlet boundary condition), and thus acts as a heat sink. Convection and radiation effects can be neglected and were not taken into account. The simulated temperatures that are presented in the remainder of this paper are extracted at $r=0.45 D$ at half thickness of the III-V membrane since the thermal dependence of the lasing characteristics is determined by the temperature at the location of the laser mode (the dot in Fig. 4). The heat is assumed to be dissipated homogeneously over the entire microdisk volume, and therefore, a total dissipated power $P$ results in $q_{v}=P /\left(\pi R^{2} d\right)$, with $R$ being the radius and $d$ being the thickness of the disk. The material parameters that were used in the simulation are listed in Table I. $\mathrm{SiO}_{2}$ films can have a thermal conductivity $\kappa$ in the range of 0.8-1.4 W/mK, depending on the deposition technique [13]. Thermally grown $\mathrm{SiO}_{2}$ films have $\kappa=1.27 \mathrm{~W} / \mathrm{mK}$, whereas films that are deposited by chemical vapor deposition have $\kappa \sim 1 \mathrm{~W} / \mathrm{mK}$. For the simulations, $\kappa_{\mathrm{SiO}_{2}}=1.27 \mathrm{~W} / \mathrm{mK}$ was assumed, unless specified otherwise. The thermal conductivity of $\mathrm{Au}$ was set to half its bulk value since the evaporated $\mathrm{Au}$ layer thicknesses were about $150 \mathrm{~nm}$, and it is known that the thermal conductivity of Au decreases with decreasing film thickness [14]. For InP, the thermal conductivity was set to $40 \mathrm{~W} / \mathrm{mK}$, due to its high doping level.

The turn-on temperature evolution in a microdisk with $D=6 \mu \mathrm{m}$ was simulated for a dissipated power of 6,9 , and $15 \mathrm{~mW}$. A dissipated power of $6 \mathrm{~mW}$ is equivalent to a voltage of $7 \mathrm{~V}$ and a current of $0.85 \mathrm{~mA}\left(3 \mathrm{kA} / \mathrm{cm}^{2}\right)$, assuming that all power is dissipated as heat. The heat sink temperature was $15{ }^{\circ} \mathrm{C}$. The results are shown in [Fig. 5 (black solid lines)]. From these results, thermal resistance $R_{\mathrm{th}}=11.5 \mathrm{~K} / \mathrm{mW}$ could be extracted. The heating transient behavior can be roughly fitted by an exponential heating curve, assuming a thermal time constant of $1.67 \mu \mathrm{s}$. The thermal resistance was calculated for disk diameters in the range of $2-10 \mu \mathrm{m}$ and is inversely proportional to the disk diameter: $R_{\mathrm{th}} \sim D^{-1.15}$ (see Fig. 6). The thermal resistance decreases slower with disk diameter as compared to the increase in disk area. Thus, we can expect a better thermal behavior for smaller devices, assuming that the threshold power density for lasing does not depend on disk diameter. This is due to the fact that the heat flow at the edges

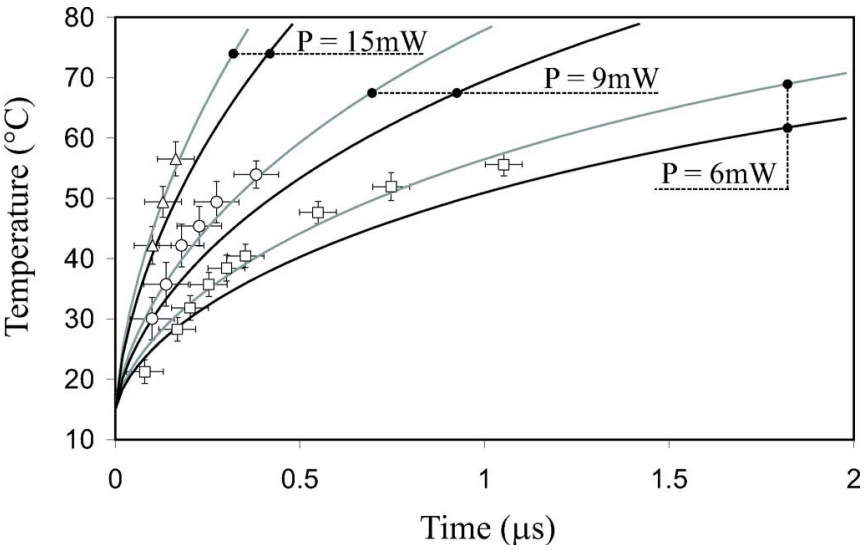

Fig. 5. Simulated turn-on temperature response for $D=6 \mu \mathrm{m}$ and dissipated powers of 6,9 , and $15 \mathrm{~mW}$ with a heat sink temperature of $15^{\circ} \mathrm{C}$, together with the experimental results for (squares) $6 \mathrm{~mW}$, (circles) $9 \mathrm{~mW}$, and (triangles) $15 \mathrm{~mW}$. The gray solid lines show the simulated response for $\kappa_{\mathrm{ox}}=1 \mathrm{~W} / \mathrm{mK}$ and provide a better fit.

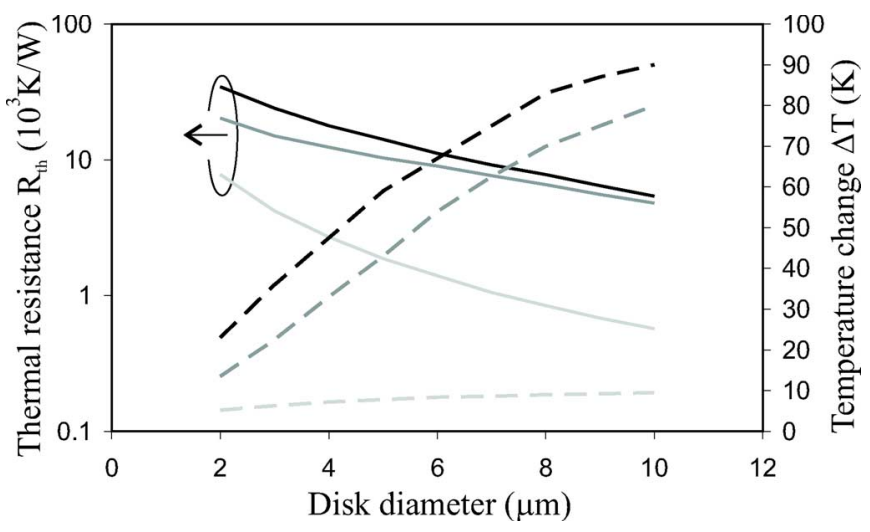

Fig. 6. (Solid lines) Thermal resistance versus disk diameter. The results for the structure in Fig. 4 are in black, the results for BCB that is replaced by amorphous $\mathrm{Al}_{2} \mathrm{O}_{3}$ are in dark gray, and the results for $\mathrm{SiO}_{2}$ that is replaced by crystalline $\mathrm{Al}_{2} \mathrm{O}_{3}$ are in light gray. Dashed lines represent temperature increase for $21-\mathrm{kW} / \mathrm{cm}^{2}$ dissipation.

of the disk becomes more dominant for smaller disk diameters. The thin lateral bottom contact layer indeed plays an important role in the heat evacuation for these smaller microdisks. To illustrate this, the expected temperature increase $\Delta T=R_{\mathrm{th}} P$ due to self-heating is plotted as a function of disk diameter, assuming a constant power dissipation of $3 \mathrm{kA} / \mathrm{cm}^{2} \times 7 \mathrm{~V}=$ $21 \mathrm{~kW} / \mathrm{cm}^{2}$ (see Fig. 6). For $D=2 \mu \mathrm{m}, \Delta T$ is reduced to $23 \mathrm{~K}$. With the heat sink at $15^{\circ} \mathrm{C}$, the device temperature should remain below $40{ }^{\circ} \mathrm{C}$, allowing $\mathrm{CW}$ lasing.

The thermal resistance can be decreased by improving the heat flow through the bonding layer. Reducing the bondinglayer thickness improves heat evacuation, but bonding layers that are too thin will cause optical substrate leakage. Another option is to use a bonding material with better thermal conductivity and low refractive index, such as crystalline $\mathrm{Al}_{2} \mathrm{O}_{3}$ or microcrystalline $\mathrm{MgO}$ (see Table I, [15], and [16]). By replacing $\mathrm{SiO}_{2}$ with $\mathrm{Al}_{2} \mathrm{O}_{3}$, the thermal resistance can be reduced by almost one order of magnitude [Fig. 6 (light gray lines)]. The temperature increase remains for all disk diameters under $10 \mathrm{~K}$. However, bonding with (crystalline) $\mathrm{Al}_{2} \mathrm{O}_{3}$ layers is technologically far more challenging than that with $\mathrm{SiO}_{2}$. An alternative 


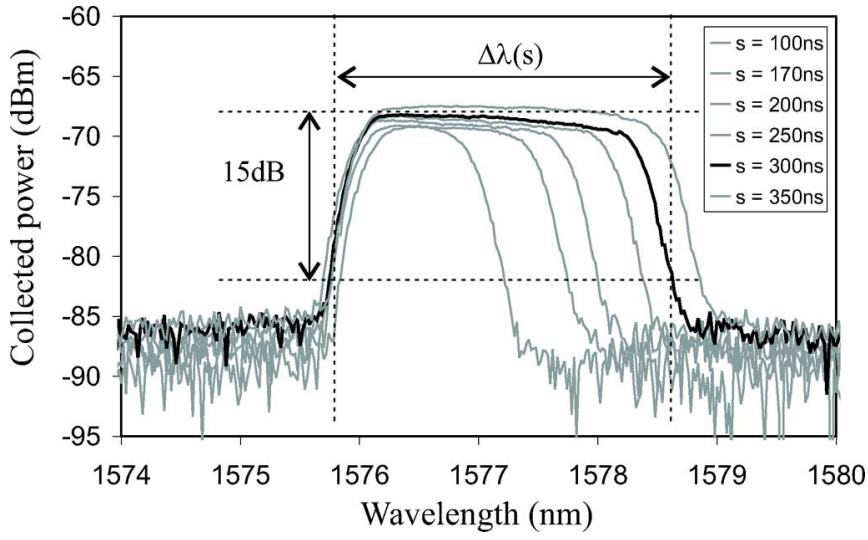

Fig. 7. Time-averaged lasing spectrum for a microdisk laser with $D=6 \mu \mathrm{m}$ versus pulse duration, with a fixed period of $5 \mu \mathrm{s}$. The spectral broadening due to self-heating is extracted at $-15,-10$, and $-5 \mathrm{~dB}$ of the peak power.

strategy is to spread the heat over a bigger area so that the heat flows through a bigger part of the bonding layer. This effect is already present due to the thin lateral InP contact layer but can be greatly enhanced if the $\mathrm{BCB}$ planarization layer is replaced with a better thermal conductor. It is highly unlikely that crystalline $\mathrm{Al}_{2} \mathrm{O}_{3}$ can be used as a planarization layer since it needs to be deposited; therefore, we assumed to have amorphous $\mathrm{Al}_{2} \mathrm{O}_{3}$ in this case $(\kappa=2 \mathrm{~W} / \mathrm{Km})$. The thermal resistance for structures where the $\mathrm{BCB}$ is replaced by amorphous $\mathrm{Al}_{2} \mathrm{O}_{3}$ and with $\mathrm{a} \mathrm{SiO}_{2}$ bonding layer is also shown in [Fig. 6 (dark gray lines)]. The thermal resistance is reduced by $10 \%-40 \%$, depending on the disk diameter. However, bigger reduction might be needed to achieve $\mathrm{CW}$ lasing in structures with high electrical resistance, particularly, for large microdisk diameters. Finally, another solution can be used to create a thermal short circuit between the disk volume and the Si substrate by etching a hole through the bonding layer and depositing good thermal (metal) connection between the Si substrate and the microdisk.

\section{EXPERIMENTAL THERMAL CHARACTERIZATION}

In order to test our simulation results, we extracted the heating and cooling behavior of the microdisk lasers by inspecting the laser emission spectrum as a function of pulse drive parameters. As the microdisk temperature increases due to selfheating, the emission wavelength shifts to longer wavelengths at a rate $d \lambda_{r} / d T$. This effect is visible on the recorded spectra as a broadening of the laser peak since the spectrum analyzer integrates the collected optical power over a time period that is much longer than the thermal time constant.

In the first set of measurements, the pulse period was set to a fixed value $(5 \mu \mathrm{s})$, which is long enough to allow the device to cool down between current pulses. Then, the emission spectrum was measured as function of pulse duration $s$. The spectra for a device with $D=6 \mu \mathrm{m}$ are shown in Fig. 7. The left edge of the laser peak can be found at approximately the same wavelength for all pulse durations. The right edges, however, show an increasing red shift with increasing pulse duration. For each spectrum, the spectral broadening $\Delta \lambda(s)$ was measured at $-15,-10$, and $-5 \mathrm{~dB}$ from the peak power. For each of these

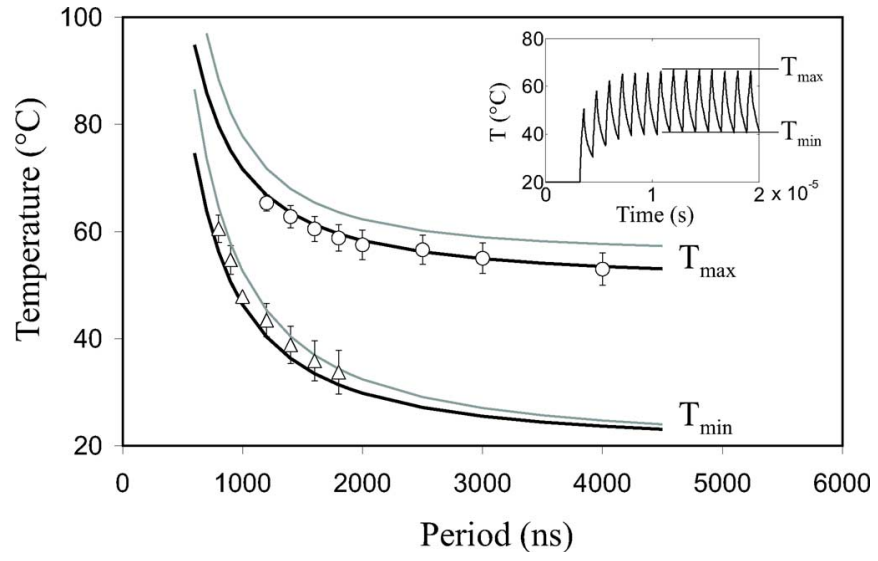

Fig. 8. Minimum and maximum pulsed-regime temperatures versus period with a fixed pulse duration (360 ns) and 11-mW dissipated power. The solid lines are the simulation results $\left(\kappa_{\text {ox }}=1.27 \mathrm{~W} / \mathrm{mK}\right.$ in black and $\kappa_{\text {ox }}=$ $1 \mathrm{~W} / \mathrm{mK}$ in gray), and the circles and triangles are the measurements. The heat sink temperature is $20^{\circ} \mathrm{C}$.

measurement sets, maximum temperature $T(s)$ at the disk edge was then estimated using

$$
T(s)=T_{\mathrm{hs}}+\frac{\Delta \lambda(s)-\Delta \lambda_{0}}{d \lambda_{r} / d T}
$$

where $T_{\mathrm{hs}}$ is the heat sink temperature, $d \lambda_{r} / d T=86 \mathrm{pm} / \mathrm{K}$, and $\Delta \lambda_{0}$ is the spectral broadening due to the limited resolution of our measurement setup, which depends on the power level at which the linewidth is extracted. For a resolution of $0.2 \mathrm{~nm}$, we get $\Delta \lambda_{0}=1.11 \mathrm{~nm}$ at $-15 \mathrm{~dB}$, assuming a Lorentzian line shape. These experimental results are compared with the simulated thermal turn-on response in Fig. 5 for $D=6 \mu \mathrm{m}$ and dissipated powers of 6,9 , and $15 \mathrm{~mW}$. The horizontal error bars show the uncertainty on the pulsewidths due to electrical loading effects, whereas the vertical error bars show the variation on the measurement that results from extracting the spectral broadening at different power levels. The experimental results show a slightly higher device temperature than the simulations with $\kappa_{\mathrm{SiO}_{2}}=1.27 \mathrm{~W} / \mathrm{mK}$ predict. Decreasing $\kappa_{\mathrm{SiO}_{2}}$ to $1 \mathrm{~W} / \mathrm{mK}$ gives a better fit.

In the second set of measurements, the pulse duration was fixed at $360 \mathrm{~ns}$, and the period was varied. For shorter periods, the device does not get the time to cool down to the heat sink temperature, and heat gradually accumulates at each period. In the regime, the temperature will oscillate between minimum temperature $T_{\min }$ and maximum temperature $T_{\max }$, which are both dependent on the period. Thus, this measurement also incorporates the cooling behavior of the device. The results for a device with $D=7 \mu \mathrm{m}, P=11 \mathrm{~mW}$, and $T_{\mathrm{hs}}=20{ }^{\circ} \mathrm{C}$ are shown in Fig. 8. For $T>65{ }^{\circ} \mathrm{C}$, the lasing signal was too weak to do proper spectral inspection. For $T<30{ }^{\circ} \mathrm{C}$, there was mode competition with a higher order mode (at shorter wavelength), which prevented a straightforward extraction of the minimum regime temperature. In this case, experimental results agree slightly better with $\kappa_{\mathrm{SiO}_{2}}=1.27 \mathrm{~W} / \mathrm{mK}$ than with $\kappa_{\mathrm{SiO}_{2}}=1 \mathrm{~W} / \mathrm{mK}$. Based on these two experiments, we conclude that the $\mathrm{SiO}_{2}$ film has a thermal conductance in the range of $1-1.27 \mathrm{~W} / \mathrm{mK}$, which is consistent with its PECVD-nature. 


\section{CONCLUSION}

We have performed a numerical and experimental analysis of the thermal behavior of electrically injected microdisk lasers that are defined in an InGaAsP-based thin film bonded on top of a silicon wafer, incorporating a $\mathrm{BCB}$ isolation and planarization layer. Lasing at room temperature was only possible in pulsed regime due to self-heating effects. Simulation results indicated that the thermal resistance is on the order of $10^{4} \mathrm{~K} / \mathrm{W}$. The thermal behavior can be improved by using a material with a higher thermal conductivity, such as $\mathrm{Al}_{2} \mathrm{O}_{3}$, for the bonding layer and/or the isolation layer. The thermal turn-on response and pulsed-regime temperatures were experimentally extracted by inspecting thermal spectral broadening, and the results were found to be in good agreement with simulation results.

\section{ACKNOWLEDGMENT}

The authors would like to thank S. Verstuyft for the III-V processing of the devices.

\section{REFERENCES}

[1] T. Baba, M. Fujita, A. Sakai, M. Kihara, and R. Watanabe, "Lasing characteristics of GaInAsP-InP strained quantum-well microdisk injection lasers with diameter of 2-10 $\mu \mathrm{m}$," IEEE Photon. Technol. Lett., vol. 9, no. 7, pp. 878-880, Jul. 1997.

[2] S. M. K. Thiyagarajan, D. A. Cohen, A. F. J. Levi, S. Ryu, R. Li, and P. D. Dapkus, "Continuous room-temperature operation of microdisk laser diodes," Electron. Lett., vol. 35, no. 15, pp. 1252-1254, Jul. 1999.

[3] R. Ushigome, M. Fujita, A. Sakai, T. Baba, and Y. K. Kubun, "GaInAsP microdisk injection laser with benzocyclobutene polymer cladding and its athermal effect," Jpn. J. Appl. Phys., vol. 41, no. 11A, pp. 6364-6369, Nov. 2002.

[4] M. Fujita, R. Ushigome, and T. Baba, "Continuous wave lasing in GaInAsP microdisk injection laser with threshold current of $40 \mu \mathrm{A}$," Electron. Lett., vol. 36, no. 9, pp. 790-791, Apr. 2000.

[5] S. J. Choi, K. Djordjev, S. J. Choi, and P. D. Dapkus, "Microdisk lasers vertically coupled to output waveguides," IEEE Photon. Technol. Lett., vol. 15, no. 10, pp. 1330-1332, Oct. 2003.

[6] W. Bogaerts, R. Baets, P. Dumon, V. Wiaux, S. Beckx, D. Taillaert, B. Luyssaert, J. Van Campenhout, P. Bienstman, and D. Van Thourhout, "Nanophotonic waveguides in silicon-on-insulator fabricated with CMOS technology," J. Lightw. Technol., vol. 23, no. 1, pp. 401-412, Jan. 2005.

[7] O. Boyraz and B. Jalali, "Demonstration of a silicon Raman laser," Opt. Express, vol. 12, no. 21, pp. 5269-5273, Oct. 2004.

[8] H. S. Rong, A. S. Liu, R. Jones, O. Cohen, D. Hak, R. Nicolaescu, A. Fang, and M. Paniccia, "An all-silicon Raman laser," Nature, vol. 433, no. 7023, pp. 292-294, Jan. 2005.

[9] C. Seassal, P. Rojo-Romeo, X. Letartre, P. Viktorovitch, G. Hollinger, E. Jalaguier, S. Pocas, and B. Aspar, "InP microdisk lasers on silicon wafer: CW room temperature operation at $1.6 \mu \mathrm{m}$," Electron. Lett., vol. 37, no. 4, pp. 222-223, Feb. 2001.

[10] H. T. Hattori, C. Seassal, E. Touraille, P. Rojo-Romeo, X. Letartre, G. Hollinger, P. Viktorovitch, L. Di Cioccio, M. Zussy, L. El Melhaoui, and J. M. Fedeli, "Heterogeneous integration of microdisk lasers on silicon strip waveguides for optical interconnects," IEEE Photon. Technol. Lett., vol. 18, no. 1, pp. 223-225, Jan. 2006.

[11] H. Park, A. W. Fang, S. Kodama, and J. E. Bowers, "Hybrid silicon evanescent laser fabricated with a silicon waveguide and III-V offset quantum well," Opt Express, vol. 13, no. 23, pp. 9460-9464, Nov. 2005.

[12] P. Rojo-Romeo, J. Van Campenhout, P. Regreny, A. Kazmierczak, C. Seassal, X. Letartre, G. Hollinger, D. Van Thourhout, R. Baets, M. Fedeli, and L. Di Cioccio, "Heterogeneous integration of electrically driven microdisk based laser sources for optical interconnects and photonic ICs," Opt Express, vol. 14, no. 9, pp. 3864-3871, May 2006.

[13] M. G. Burzo, P. L. Komarov, and P. E. Raad, "Thermal transport properties of gold-covered thin-film silicon dioxide," IEEE Trans. Compon. Packag. Technol., vol. 26, no. 1, pp. 80-88, Mar. 2003.
[14] G. Chen and P. Hui, "Thermal conductivities of evaporated gold films on silicon and glass," Appl. Phys. Lett., vol. 74, no. 20, pp. 2942-2944, May 1999.

[15] S. M. K. Thiyagarajan, A. F. J. Levi, C. K. Lin, I. Kim, P. D. Dapkus, and S. J. Pearton, "Continuous room-temperature operation of optically pumped InGaAs/InGaAsP microdisk lasers," Electron. Lett., vol. 34, no. 24, pp. 2333-2334, Nov. 1998.

[16] S. M. Lee, D. G. Cahill, and T. H. Allen, "Thermal-conductivity of sputtered oxide-films," Phys. Rev. B, Condens. Matter, vol. 52, no. 1, pp. 253 257, Jul. 1995.

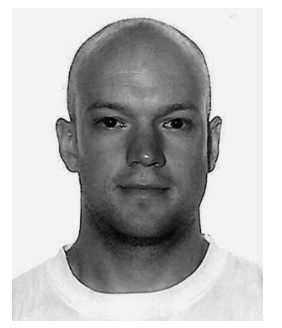

Joris Van Campenhout ( $\left.\mathrm{S}^{\prime} 01\right)$ received the M.S. degree in physics engineering from Ghent University, Ghent, Belgium, in 2002. He is currently working toward the Ph.D. degree in electrical engineering at the Photonics Research Group, Department of Information Technology, Ghent University-Interuniversity Microelectronics Centre.

His research interests include the design, fabrication, and characterization of silicon-based photonic integrated circuits. Over the past few years, he has been working on the heterogeneous integration of InP-based microcavity lasers-based on photonic-crystal and microdisk structures—on a silicon platform.

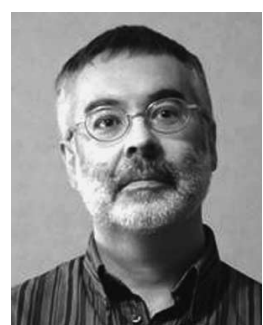

Pedro Rojo-Romeo was born in Madrid, Spain, in 1958. He received the Dipl.Ing. degree from the Institut National des Sciences Appliques, Lyon, France, in 1981 and the Ph.D. degree in electronic devices from Institut National des Sciences Appliques, in 1984.

$\mathrm{He}$ is currently an Associate Professor of electronics with the Laboratoire d'Electronique, Microelectronique et Micro-systemes, Ecole Centrale de Lyon-Center National de la Recherche Scientifique, Ecully, France. His primary research interests include electrical and optical device fabrication technology and characterization of microelectronic and optoelectronic micronanodevices. He is also involved in optical interconnection systems, nanotechnologies, and optical integrated circuits technologies.

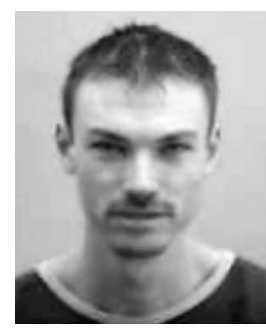

Dries Van Thourhout (M'98) received the M.S degree in physical engineering and the Ph.D. degree from Ghent University, Ghent, Belgium, in 1995 and 2000 , respectively.

From October 2000 to September 2002, he was with Lucent Technologies, Bell Laboratories, Murray Hill, NJ, working on the design, processing, and characterization of $\mathrm{InP} / \mathrm{InGaAsP}$ monolithically integrated devices. In October 2002, he joined the Department of Information Technology (INTEC), Ghent University, where he is currently a member of the permanent staff of the Photonics Group. He is a Lecturer and Co-Lecturer of four courses within the Ghent University Master in Photonics program (Microphotonics, Advanced Photonics Laboratory, Photonic Semiconductor Components, and Technology). He is also coordinating the cleanroom activities of the research group. He has authored and coauthored more than 30 journal papers and has presented invited papers at several major conferences. He is the holder of three patents. His research interests include the design, fabrication, and characterization of integrated photonic devices. Main topics involve silicon nanophotonic devices, heterogeneous integration of InP-on-silicon, and integrated InP-based optical isolators. He is also working on the development of new fabrication processes for photonic devices, e.g., based on focused ion beam etching and die-to-wafer bonding. 


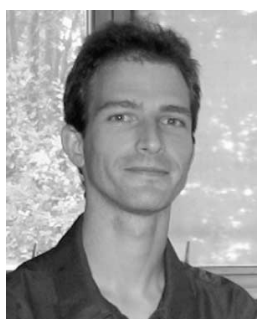

Christian Seassal received the B.Eng. degree from the Institut National des Sciences Appliquees de Lyon (INSA), Villeurbanne, France, in 1993 and the $\mathrm{Ph} . \mathrm{D}$. degree in condensed matter from the Ecole Centrale de Lyon (ECL), Ecully, France, in 1997.

Since 1998, he has been a Center National de la Recherche Scientifique (CNRS) Research Scientist is currently with the Nanophotonics Group, Institut des Nanotechnologies de Lyon, ECL (INL, which is a joint research unit between CNRS, ECL, INSA, and the University of Lyon). He is the author and coauthor of approximately 50 papers in international journals and 70 international conference proceedings. His research interests include active integrated micronanophotonic structures and MOEMS. His recent experiments deal with photonic-crystal lasers and nonlinear devices, quantum-dot nanophotonic devices, heterogeneous integration between active III-V photonic devices, and silicon photonic circuits.

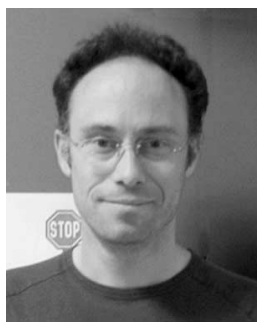

Philippe Regreny was born in France in $1967 . \mathrm{He}$ received the M.S. degree in materials science from Rennes University, Rennes, France, and the Ph.D. degree in electronics from the Ecole Centrale de Lyon, Ecully, France, in 1997.

He is currently a Research Engineer with the Laboratoire d'Electronique, Microelectronique et MicroSystemes, Ecole Centrale de Lyon-Center National de la Recherche Scientifique, Ecully. His current research interests include epitaxial growth of III-V semiconductor compounds and their integration on silicon.

Lea Di Cioccio received the degree in physics engineering from the Institut $\mathrm{Na}$ tional des Sciences Appliquees, Rennes, France, the M.S. degree in metallurgy and material science from Paris VI University, Paris, France, in 1985, and the $\mathrm{Ph} . \mathrm{D}$. degree in material and semiconductor physics from the Institut National Polytechnique de Grenoble, Grenoble, France, in 1988.

In 1990, she joined the Commissariat a l'Energie Atomique/Laboratoire d'Electronique de Technologie de l'Information, Grenoble, where she is currently a Specialist in semiconductor material heterostructures and 3-D integration using various processes such as epitaxy, wafer bonding, and the Smart CutTM process. She has engaged in characterization such as transmission electron microscopy. She is the author and coauthor of more than 100 publications. She is the holder of 15 patents.

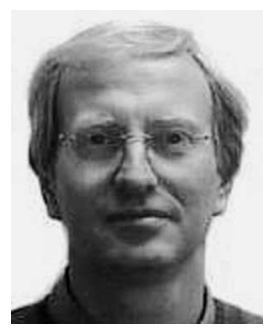

Jean-Marc Fedeli received the electronics engineer diploma from the Institut National Polytechnique de Grenoble, Grenoble, France, in 1978.

$\mathrm{He}$ then conducted research at the Commissariat a l'Energie Atomique/Laboratoire d'Electronique de Technologie de l'Information (CEA/LETI), Grenoble, on various magnetic memories and magnetic components as a Project Leader, Group Leader, and Program Manager. For two years, he was an Advanced Program Director with MEMSCAP for the development of RF MEMS. In 2002, he then returned to CEA/LETI as a Coordinator of silicon photonic projects. Under a large partnership with universities and research laboratories, he works on various technological aspects on photonics on CMOS ( $\mathrm{Si} \mathrm{rib}$ and stripe waveguides, and Si3N4 and a-Si waveguides), Si modulators, Ge photodetectors, SiOx material, and InP sources on Si. His main focus is on the integration of a photonic layer at the metallization level of an electronic circuit.

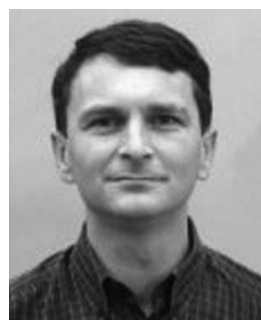

Roel Baets (M'88-SM'96-F'07) received the degree in electrical engineering from Ghent University, Ghent, Belgium, in 1980, the M.Sc. degree in electrical engineering from Stanford University, Stanford, CA, in 1981, and the Ph.D. degree from Ghent University in 1984

Since 1981, he has been with the Department of Information Technology (INTEC), Ghent University. Since 1989, he has been a Professor with the engineering faculty of Ghent University. From 1990 to 1994, he was a Part-Time Professor at the Technical University of Delft, Delft, The Netherlands. Since 2004, he has been a PartTime Professor at the Technical University of Eindhoven, Eindhoven, The Netherlands. He currently leads the Photonics Research Group, INTEC, Ghent University (an associated lab of IMEC), which focuses on new concepts for photonic components and circuits for optical communication, optical interconnect, and optical sensing. He has mainly worked in the field of photonic components. He has been involved in various European research projects and has been the Coordinator of some of them. He currently coordinates the European Network of Excellence ePIXnet. With about 200 journal publications and 400 conference proceedings papers as well as about ten patents, he has made contributions to research on III-V semiconductor laser diodes, passive guided wave, and grating devices, and to the design and fabrication of photonic ICs

Prof. Baets is a member of the Optical Society of America, the IEEE Lasers and Electro-Optics Society (LEOS), The International Society for Optical Engineers, and the Flemish Engineers Association. He has been a member of the program committees of a.o. OFC, ECOC, the IEEE Semiconductor Laser Conference, ESSDERC, CLEO-Europe, the LEOS Annual Meeting, Photonics Europe, and ECIO. He was the Chairman of the IEEE-LEOS-Benelux Chapter from 1999 to 2001. From 2003 to 2005, he was an elected Member of the Board of Governors of the IEEE LEOS. 